\title{
Actitud de los alumnos de quinto de medicina hacia la salud mental
}

\author{
Inmaculada Gilaberte, Inmaculada Failde, Alejandro Salazar, Luis Caballero
}

Introducción. El estigma social que persiste hacia la enfermedad mental puede estar presente en los estudiantes de medicina e impactar en la forma de considerar ésta y la psiquiatría como especialidad.

Objetivo. Investigar la actitud hacia la enfermedad mental y la psiquiatría en una muestra de estudiantes.

Sujetos y métodos. Se realizó una encuesta estructurada, voluntaria y anónima a los 27 estudiantes de quinto curso del grado de medicina de la Universidad San Pablo-CEU de Madrid. La encuesta incluía un cuestionario sobre el nivel de familiaridad hacia la enfermedad mental, el cuestionario de actitud hacia la enfermedad mental (AMI) y el cuestionario adaptado de Balon sobre la actitud hacia la psiquiatría.

Resultados. Un total de 22 alumnos respondió la encuesta (72,7\% mujeres). Estos alumnos presentaron una alta familiaridad con la enfermedad mental (puntuación media: 7,27; máximo: 11). El 81,8\% refirieron una actitud positiva hacia la enfermedad mental (escala AMI). Las puntuaciones de la escala de Balon también mostraron un nivel alto de acuerdo en los méritos globales de la psiquiatría y en la eficacia de ésta. Tampoco las respuestas respecto al rol y función del psiquiatra y a la carrera profesional revelaron una postura negativa. No hubo relación entre la familiaridad y la actitud hacia la enfermedad mental o hacia la psiquiatría.

Conclusiones. En esta muestra no se evidencia la existencia de estigma hacia la enfermedad mental o hacia la psiquiatría. Posteriores estudios en muestras más amplias y evaluaciones de la enseñanza de la psiquiatría son necesarios para profundizar en este tema.

Palabras clave. Enfermedad mental. Estigma. Estudiantes de medicina.

\section{Medicine 5th course student's attitudes towards mental health}

Introduction. Medicine students could share that stigmatizing attitudes towards mental illness and Psychiatry as specialty. Aim. To investigate the attitudes of medical students towards the mental disease and the psychiatry.

Subjects and methods. 27 medicine 5th course students from San Pablo-CEU University were interviewed with a structured interview containing questionnaires of familiarity with mental disease, attitudes towards mental disease and the Balon's adapted questionnaire to investigate the positive and negative views towards psychiatry as specialty.

Results. 22 students responded the interview (72.7\% women). The students showed a high familiarity regarding mental disease (mean score: 7.27; max.: 11). 81.8\% of the students showed positive attitudes towards mental disease according with the AMI questionnaire. The students' attitudes and views of psychiatry were mostly positive with heterogeneous agreement on the role of psychiatrists and the career rewards. We don't find any relation between familiarity regarding mental disease and the attitude to these pathologies and the psychiatry as a professional specialty.

Conclusions. Students' attitudes towards mental illness and psychiatry didn't reveal any stigmatized attitudes. More studies with larger samples are needed to know more deeply the students attitudes and if medical education could influence them.

Key words. Medicine students. Mental disease. Stigma.

\section{Introducción}

Es bien conocido el estigma social que sigue existiendo hacia las personas que sufren una enfermedad mental [1]. Este estigma parece subsistir también dentro de los profesionales sanitarios. Algunos autores han mostrado que este hecho puede influir en el retraso en el diagnóstico de estas enfermedades, o en el hecho contrastado de que los pacientes con enfermedades mentales reciban una peor atención en otras patologías que padezcan [2].

La mayoría de las investigaciones que se han realizado sobre el estigma se han focalizado en la actitud que hacia la enfermedad mental tiene la socie-
Universidad de Cádiz (I. Failde, A. Salazar). Universidad San Pablo-CEU (I. Gilaberte). Departamento de Psiquiatría; Hospital Puerta de Hierro; Universidad San Pablo-CEU (L. Caballero). Madrid, España.

Correspondencia: Dra. Inmaculada Gilaberte. Pabellón Docente. Hospital Universitario Montepríncipe. Avda. Montepríncipe, 25. E-28660 Boadilla del Monte (Madrid).

E-mail: inmagilaberte@live.com

Agradecimientos:

A todos los alumnos de quinto curso de medicina de la Universidad San Pablo-CEU, por su contribución desinteresada en este proyecto.

Conflicto de intereses: No declarado.

Conflict of interests: None declared.

(c) 2012 Educación Médica 
Tabla I. Nivel de familiaridad según el cuestionario [4].

Ítems
1. No ha observado nunca a una persona con enfermedad mental
2. Ha observado de paso a alguna persona con enfermedad mental
3. Ha visto alguna película sobre enfermedades mentales
4. Ha visto algún documental de televisión sobre enfermedades mentales
5. Ha observado con frecuencia a alguna persona con enfermedad mental
6. Ha trabajado con una persona con enfermedad mental
7. Su trabajo incluye servicios a personas con enfermedad mental
8. Un amigo de la familia padece una enfermedad mental
9. Un pariente padece una enfermedad mental
10. Vive con una persona que padece una enfermedad mental

0

0

$1(4,5 \%)$

$5(22,7 \%)$

$1(4,5 \%)$

0

$1(4,5 \%)$

$3(13,6 \%)$

$9(40,9 \%)$

$2(9,1 \%)$

11. Padece una enfermedad mental grave

0

Puntuación (media $\pm \mathrm{DE}$ )

$7,270 \pm 2,394$

Rango

3-10

dad, con estudios realizados en población general [3-5]. Estos trabajos muestran que aquellas personas que tienen más familiaridad con la enfermedad mental o han tenido algún contacto con un paciente con un trastorno psiquiátrico cambian hacia una actitud menos estigmatizadora [5].

Los estudiantes de medicina ya establecen preferencias por categorías particulares de pacientes, y según algunos estudios parece que muestran menos empatía hacia los pacientes psiquiátricos que hacia otros tipos de pacientes [6]. De hecho, la psiquiatría parece ser una de las especialidades menos atractivas para los graduados en esta profesión [7]. Se piensa que esto es así porque los estudiantes de medicina podrían estar compartiendo con la población general una actitud estigmatizante hacia la enfermedad mental y hacia la psiquiatría como especialidad, calificándola de poco científica, imprecisa e ineficaz [8].

En este estudio pretendemos investigar la actitud ante la enfermedad mental y la psiquiatría como especialidad en una muestra de estudiantes de quinto de medicina de una facultad de nuestro país, y conocer si existe una asociación entre el grado de conocimiento de estas enfermedades y la actitud hacia ellas y hacia la psiquiatría. Así mismo, queremos investigar si existe asociación entre la actitud hacia la enfermedad mental y la actitud hacia la psiquiatría como especialidad médica.

\section{Sujetos y métodos}

\section{Diseño del estudio}

El estudio tiene un diseño observacional, descriptivo y transversal.

\section{Población del estudio}

La muestra estaba constituida por los 27 alumnos que componen el quinto curso del grado de medicina de la Universidad San Pablo-CEU de Madrid. La encuesta se realizó antes de iniciar la docencia de la asignatura de psiquiatría.

\section{Instrumentos y variables del estudio}

\section{Datos sociodemográficos}

Se recogió la edad y sexo de los participantes.

\section{Cuestionario sobre el nivel de familiaridad hacia la enfermedad mental (Level of Familiarity Questionnaire) [4]}

Este cuestionario evalúa 12 situaciones que se clasifican de acuerdo a la intimidad y contacto que el entrevistado ha tenido con algún paciente que sufra enfermedad mental en orden descendente, siendo la puntuación de 0 la más baja posible, y la puntuación de 11, la mayor. El entrevistado evalúa varias situaciones y se puntúa con el índice que indique mayor familiaridad, es decir, con el valor más alto. La escala se ha validado y utilizado en varios estudios tanto de población general como de profesionales sanitarios y estudiantes en inglés. Para usar el instrumento en nuestro idioma realizamos una validación lingüística de éste mediante el método de traducción-retrotraducción, en el que la escala original fue traducida al castellano de forma independiente por dos personas bilingües. Junto con la traducción de los ítems se preguntó sobre la dificultad de encontrar una expresión en castellano conceptualmente equivalente a la original. Posteriormente, se realizó una revisión por dos psiquiatras para detectar posibles errores o imprecisiones. A continuación se efectuó la retrotraducción al inglés de las dos versiones que realizaron otros traductores diferentes que desconocían la versión original. En esta retrotraducción también se evaluaron las dificultades. Los psiquiatras realizaron una segunda revisión valorando la equivalencia con la versión original. Ninguno de los ítems resultó problemático y hubo acuerdo entre los psiquiatras en que la versión original y la traducida eran comparables. 


\section{Cuestionario sobre actitudes hacia la enfermedad mental (Attitude to Mental Illness Questionnaire) [9]} El Attitude to Mental Illness Questionnaire (AMI) tiene 20 ítems que examinan la actitud hacia las causas, tratamiento y consecuencias de la enfermedad mental y su impacto en los individuos y la sociedad. Fue creado por Singh et al [9] en 1998 para evaluar la actitud de estudiantes de medicina usando el feedback de éstos. Los ítems se puntúan según una escala tipo Likert de cinco puntos, con la puntuación más alta asociada a la actitud más favorable. La escala se ha validado en inglés.

Para validar la versión lingüística en castellano utilizamos el mismo método de traducción-retrotraducción que en el caso anterior. Sólo la traducción de los ítems 8 y 10 tuvo que ser modificada por psiquiatras expertos en esta terminología. En los ítems restantes hubo acuerdo entre los psiquiatras de que la versión original y la traducida resultaban comparables.

El cuestionario AMI se valora sumando la puntuación de los 20 ítems. Se considera una puntuación neutral en el punto de corte de 60. Por debajo de esta puntuación se considera que los entrevistados tienen una actitud negativa. Por encima de 60, la actitud se considera positiva.

\section{Cuestionario de Balon et al [10]}

Este cuestionario traducido del original de 29 preguntas, que se desarrolló para medir las actitudes de los estudiantes de medicina sobre la psiquiatría, ya se ha utilizado en una investigación previa sobre la actitud en los estudiantes de una universidad en nuestro país [11]. En él se recogen seis aspectos: méritos globales de la psiquiatría, eficacia, definición de rol y función del psiquiatra, posibles abusos y crítica social, apoyo y carrera profesional, y factores específicos de la unidad docente. Los ítems se puntúan según una escala tipo Likert de cinco, puntos con la puntuación más alta asociada a la actitud más desfavorable. No se cumplimentaron los ítems correspondientes al aspecto 'factores específicos de la unidad docente', ya que los entrevistados todavía no habían iniciado el programa docente. Por tanto, sólo se analizaron los primeros 23 ítems del cuestionario. Las respuestas se evaluaron de forma independiente.

\section{Procedimiento del estudio}

Este estudio ha sido avalado por el director de la asignatura y por el Decanato de la Facultad de Medicina de la Universidad San Pablo-CEU de Madrid.
Los profesores de la asignatura de psiquiatría pidieron el consentimiento verbal de cada alumno para participar en esta investigación al comienzo del curso y explicaron el carácter voluntario y anónimo de esta participación, garantizando la confidencialidad de los datos.

A aquellos alumnos que decidieron participar en esta investigación se les distribuyó el cuestionario en horario docente, durante la segunda clase del curso. El cuestionario se completó más tarde de forma voluntaria y se entregó en la secretaría para preservar el carácter anónimo tanto de los encuestados como del contenido por parte de los investigadores.

\section{Análisis estadístico}

Se realizó un análisis descriptivo de los resultados utilizando medidas de tendencia central (medias, medianas y desviaciones estándares) y medidas de frecuencia. Para evaluar la relación entre la familiaridad y la actitud hacia la enfermedad mental se calculó el coeficiente de correlación de Spearman, ya que no podía asumirse la distribución normal de la puntuación de la familiaridad en base a los resultados del test de normalidad de Kolmogorov-Smirnov. Para evaluar la relación entre la familiaridad y la actitud hacia la psiquiatría, así como entre esta última y la actitud hacia la enfermedad mental, se realizaron pruebas de Kruskal-Wallis para cada uno de los ítems del cuestionario de Balon.

Los datos se tabularon y analizaron mediante el programa estadístico SPSS v. 17.

\section{Resultados}

La muestra del estudio se componía de 27 estudiantes de la asignatura de psiquiatría en el grado de medicina de la Universidad San Pablo-CEU. De ellos, 22 (81,5\%) respondieron el cuestionario. El $72,7 \%$ eran mujeres. La edad media de la muestra fue de 23,05 \pm 2,43 años.

Estos alumnos presentaron una alta familiaridad con la enfermedad mental, con una puntuación de 7,27 en la escala de familiaridad que puntúa hasta un máximo de 11; destacaba que el 40,9\% de los alumnos afirmaba tener algún familiar que padecía una enfermedad mental (Tabla I).

La puntuación media en la escala AMI, de actitud hacia la enfermedad mental, fue de 69,4 $\pm 6,9$ (rango: 59-87). El 81,8\% de los alumnos tenían una actitud positiva hacia la enfermedad mental, entendiendo ésta como una puntuación total en la escala AMI $>60$. 
Las puntuaciones del la escala de Balon muestran también una actitud positiva hacia la psiquiatría. De la escala se consideraron cinco aspectos: méritos globales de la psiquiatría (preguntas 1-3), eficacia (preguntas 4-6), definición de rol y función del psiquiatra (preguntas 7-13), posibles abusos y crítica social (preguntas 14 y 15), y apoyo y carrera profesional (preguntas 16-23) (Tabla II).

En relación con los méritos globales de la psiquiatría, el 95\% de los alumnos pensaban que la investigación en el área de psiquiatría había hecho grandes avances, y un 77,3\% se mostraban en desacuerdo con la afirmación de que la psiquiatría es una especialidad no científica. Con respecto al apartado de eficacia, un 95,5\% de los alumnos mostraban su acuerdo con la eficacia del tratamiento psiquiátrico.

En la definición del rol y la función del psiquiatra hubo más dispersión en las respuestas, especialmente en la pregunta 8 , que evalúa el pensamiento de los psiquiatras, con un $23,8 \%$ de los alumnos que se mostraban en desacuerdo con que tuvieran un pensamiento lógico y claro. También en la pregunta 9, que evalúa la cualificación de trabajadores sociales y psicólogos clínicos en comparación con los psiquiatras, con un $27,3 \%$ que se mostraban completamente de acuerdo en que tenían la misma cualificación frente a un 22,7\% que se mostraban totalmente en desacuerdo.

En relación con los abusos y la crítica social, mientras que en la pregunta 14 todos los alumnos se inclinaban a que los psiquiatras no muestran abusos de poder, en la pregunta 15 hubo más dispersión en las respuestas con respecto a la remuneración económica de estos profesionales, con un $36,4 \%$ de los encuestados que se mostraban en desacuerdo con que ésta fuera similar a la de otros especialistas.

En el último apartado, en el que se valora apoyo y carrera profesional, hubo un grado de desacuerdo moderado en la pregunta 16 (prestigio de la especialidad de psiquiatría), mientras que en las preguntas 17, 18 y 19 (grado de preferencia entre las especialidades médicas) un alto porcentaje de alumnos mostraron desacuerdo en relación con el estatus social de esta especialidad, así como que se realice por profesionales sin verdadera vocación o con baja capacitación. Así mismo, un elevado porcentaje de alumnos mostraron su desacuerdo en la falta de apoyo familiar o social por la elección de esa especialidad. Por último, un 77,2\% de los alumnos se mostraban en desacuerdo con la afirmación de sentirse incómodos con enfermos psiquiátricos.

En el análisis de la asociación entre las distintas escalas, se observó que no existía relación entre las puntuaciones de la escala de familiaridad y la actitud hacia la enfermedad mental $(r=-0,02 ; p=$ $0,933)$. En la relación entre familiaridad y actitud hacia la psiquiatría, el único ítem del cuestionario de Balon en el que la asociación resultó significativa $(p=0,047)$ fue el 19 ('la psiquiatría es una especialidad que está llena de médicos de otros países, con capacitación de baja calidad'), donde los alumnos incluidos en las categorías extremas (completamente de acuerdo y completamente en desacuerdo) estaban menos familiarizados con la enfermedad mental (puntuación media en la escala de familiaridad: 4 y 5,33, respectivamente) que aquellos que tenían opiniones más moderadas (media en la escala de familiaridad: moderadamente de acuerdo, 8; moderadamente en desacuerdo, 9,5).

En la relación entre la actitud hacia la enfermedad mental y la actitud hacia la psiquiatría, sólo se observó asociación ( $p=0,014)$ en el ítem 3 ('la psiquiatría es una especialidad no científica e imprecisa'), donde los alumnos que estaban completamente en desacuerdo con la pregunta tenían una actitud más positiva hacia la enfermedad mental (media AMI: 77,5) que el resto de los estudiantes (media AMI: 66).

\section{Discusión}

Los resultados del estudio muestran que los alumnos de quinto curso del grado de medicina de la Universidad San Pablo-CEU de Madrid tienen una actitud positiva hacia la salud mental y hacia la especialidad de psiquiatría. Así mismo no ha podido establecerse una relación entre la familiaridad de los sujetos con la enfermedad mental y la actitud hacia ella y hacia la especialidad de psiquiatría. Se observó una asociación entre una actitud positiva hacia la enfermedad mental y el desacuerdo con que la psiquiatría sea una especialidad no científica e imprecisa.

Cuando una persona identifica a otro como enfermo mental sin entender lo que es la enfermedad mental o teniendo una concepción errónea de ésta, resulta frecuente que se produzca una impresión negativa hacia ese enfermo [12]; por eso, parece que el contacto formal con personas con enfermedad mental se relaciona directamente con un aumento de la empatía hacia estos enfermos y una disminución del estigma $[4,13]$. Es de señalar que la muestra de los alumnos estudiados tenía un nivel alto de familiaridad con la enfermedad mental.

En dicha muestra, más del $80 \%$ de los alumnos presentaban una actitud positiva hacia la enferme- 
Tabla II. Escala de Balon et al [10].

\begin{tabular}{|c|c|c|c|c|}
\hline \multirow{2}{*}{ Preguntas } & \multicolumn{4}{|c|}{ Porcentajes } \\
\hline & CA & MA & MD & $C D$ \\
\hline $\begin{array}{l}\text { 1. La investigación en psiquiatría ha hecho grandes pasos en el avance } \\
\text { de la atención de los trastornos mentales más importantes }\end{array}$ & 54,5 & 40,9 & 4,5 & 0 \\
\hline 2. La psiquiatría es una frontera de la medicina en rápida expansión & 40,9 & 27,3 & 31,8 & 0 \\
\hline 3. La psiquiatría es una especialidad no científica e imprecisa & 4,5 & 18,2 & 45,5 & 31,8 \\
\hline $\begin{array}{l}\text { 4. Si algún familiar estuviera muy alterado emocionalmente } \\
\text { y no mejorase recomendaría una visita al psiquiatra }\end{array}$ & 54,5 & 45,5 & 0 & 0 \\
\hline 5. La consulta psiquiátrica para pacientes médicos o quirúrgicos es, a menudo, bastante útil & 68,2 & 27,3 & 4,5 & 0 \\
\hline 6. El tratamiento psiquiátrico es bastante útil para la gente que lo recibe & 45,5 & 50,0 & 4,5 & 0 \\
\hline 7. La psiquiatría no es una rama genuina y válida de la medicina & 9,1 & 4,5 & 9,1 & 77,3 \\
\hline 8. La mayoría de los psiquiatras siguen un pensamiento lógico y claro & 23,8 & 52,4 & 23,8 & 0 \\
\hline $\begin{array}{l}\text { 9. Con escasas excepciones, los psicólogos clínicos y los trabajadores sociales están } \\
\text { igualmente calificados que los psiquiatras para tratar personas con trastornos emocionales }\end{array}$ & 27,3 & 45,5 & 4,5 & 22,7 \\
\hline 10. Entre los profesionales de la salud mental, los psiquiatras tienen más autoridad e influencia & 45,5 & 27,3 & 18,2 & 9,1 \\
\hline $\begin{array}{l}\text { 11. Los psiquiatras, cuando enseñan la asignatura de psiquiatría, } \\
\text { demasiado a menudo piden disculpas por cómo es la especialidad }\end{array}$ & 9,1 & 27,3 & 40,9 & 22,7 \\
\hline $\begin{array}{l}\text { 12. La psiquiatría es demasiado biológica y no presta atención } \\
\text { suficiente a los problemas emocionales y de la vida del paciente }\end{array}$ & 4,5 & 13,6 & 27,3 & 54,5 \\
\hline $\begin{array}{l}\text { 13. La psiquiatría es demasiado analítica, teórica y psicodinámica, } \\
\text { y no presta atención suficiente a la fisiología del paciente }\end{array}$ & 0 & 22,7 & 45,5 & 31,8 \\
\hline $\begin{array}{l}\text { 14. Los psiquiatras abusan a menudo de su poder legal } \\
\text { para hospitalizar pacientes en contra de su voluntad }\end{array}$ & 0 & 0 & 50,0 & 50,0 \\
\hline 15. En promedio, el psiquiatra gana el mismo dinero que la mayor parte del resto de los especialistas & 4,5 & 59,1 & 36,4 & 0 \\
\hline 16. La psiquiatría tiene un bajo prestigio entre el público en general & 18,2 & 54,5 & 22,7 & 4,5 \\
\hline 17. La psiquiatría tiene un alto estatus entre las otras disciplinas médicas & 0 & 13,6 & 59,1 & 27,3 \\
\hline $\begin{array}{l}\text { 18. Muchos médicos que no pueden obtener una plaza } \\
\text { de MIR en otras especialidades entran eventualmente en psiquiatría }\end{array}$ & 0 & 22,7 & 54,5 & 22,7 \\
\hline 19. La psiquiatría es una especialidad llena de médicos de otros países, con capacitación de baja calidad & 4,5 & 9,1 & 59,1 & 27,3 \\
\hline 20. Mi familia me saca de la cabeza hacer psiquiatría & 9,1 & 0 & 22,7 & 68,2 \\
\hline 21. Mis amigos y médicos conocidos me sacan de la cabeza hacer psiquiatría & 13,6 & 4,5 & 31,8 & 50,0 \\
\hline $\begin{array}{l}\text { 22. Si un estudiante expresa interés por la psiquiatría corre } \\
\text { el riesgo de ser visto como raro, peculiar o neurótico }\end{array}$ & 9,1 & 18,2 & 36,4 & 36,4 \\
\hline 23. Me siento incómodo con enfermos psiquiátricos & 9,1 & 13,6 & 54,5 & 22,7 \\
\hline
\end{tabular}

CA: completamente de acuerdo; MA: moderadamente de acuerdo; MD: moderadamente en desacuerdo; CD: completamente en desacuerdo. 
dad mental. Este porcentaje es similar al encontrado recientemente por otros autores en alumnos de medicina [14] y señalan una ausencia de estigma hacia estas patologías.

En esta muestra, similar a otras que han evaluado estudiantes de medicina [11], la mayoría de los alumnos eran mujeres. Esta alta prevalencia femenina podría influir en la actitud hacia la enfermedad mental. Estudios previos han señalado que los hombres tienen una percepción más negativa de la enfermedad mental y que las mujeres eran menos propensas a mostrar prejuicios o discriminación hacia personas con enfermedad mental [12]. Esta menor estigmatización de la enfermedad mental por parte de las mujeres podría estar mediada por su mayor empatía social [15]. Debido al pequeño número de varones en nuestra muestra $(n=6)$, no pudimos realizar un análisis por sexo de la actitud hacia la enfermedad mental, siendo ésta una de las limitaciones de este trabajo.

La educación es otro factor que influye en el estigma. Las personas que completan más años de educación tienen también una menor propensión a estigmatizar la enfermedad mental por su conocimiento de ésta [16]. En este sentido, los estudiantes de medicina tienen un alto grado de educación que condiciona probablemente su actitud hacia la enfermedad mental, a pesar de no haber iniciado todavía el aprendizaje de la psiquiatría que, sin duda, influirá en aumentar este conocimiento [17].

Sin embargo, pensamos que la actitud positiva encontrada no está exenta de sesgos. Dos factores pueden estar sesgando este resultado; en primer lugar, una limitación de este artículo es considerar la enfermedad mental como una generalización, aunque algunas publicaciones han encontrado diferencias notables entre la actitud mostrada ante enfermedades mentales tan distintas como la depresión o la esquizofrenia $[18,19]$. Un segundo factor que puede influir es la 'deseabilidad social': aunque las encuestas fueron anónimas, el deseo de 'quedar bien' con el entrevistador, el nuevo profesor de la asignatura de psiquiatría, y la tendencia a atribuirse uno mismo actitudes socialmente deseables pueden constituir un sesgo en la valoración de este resultado [20].

Los alumnos tienen una imagen de la psiquiatría como una especialidad científica y la mayoría piensa que la investigación en esta rama de la medicina ha avanzado mucho en los últimos años. La opinión positiva sobre utilidad y eficacia del tratamiento psiquiátrico también es unánime entre los alumnos estudiados. Estos resultados son similares a los obtenidos en la Universitat Autònoma de Barcelona en el año 2005 [21].
Sin embargo, todavía se mantienen ciertas dudas sobre la función actual del médico psiquiatra y si su papel se puede superponer con el de psicólogos clínicos o trabajadores sociales. Aunque no perciben una presión social negativa hacia la elección de esta especialidad, los alumnos creen que es una especialidad que carece de un alto estatus social. Por último, la mayoría de los alumnos declaran no sentirse incómodos ante los pacientes con enfermedad mental.

Estas respuestas son comparables a las que obtuvieron Balon et al en un estudio realizado hace más de diez años en diversas universidades estadounidenses, y que mostraba que la percepción de la psiquiatría había mejorado de forma considerable desde los años ochenta [10], y a las que se han obtenido aquí en España en estudios similares realizados con un intervalo de ocho años $[11,19]$.

En esta muestra, en contraste con la mayoría de las publicaciones $[4,13]$, no encontramos que hubiera una asociación entre la alta familiaridad de los alumnos respecto a la enfermedad mental y la actitud hacia ésta y hacia la psiquiatría, aunque debido al bajo número de sujetos entrevistados no podemos concluir que no exista dicha relación en la población estudiada. Sin embargo, aquellos pacientes que mostraban una actitud positiva hacia la enfermedad mental estaban totalmente en desacuerdo con la afirmación de que la psiquiatría era una especialidad no científica. Aunque esta relación deberá confirmarse en otros estudios, parece que la falta de estigma hacia la enfermedad mental podría mejorar la actitud hacia la psiquiatría como especialidad médica.

Aunque el pequeño tamaño muestral nos hace ser cautos en nuestras conclusiones, pensamos que este estudio sugiere que la actitud de estos alumnos de medicina hacia la enfermedad mental y hacia la psiquiatría como especialidad no traduce un estigma social hacia éstas. Otros estudios con muestras más amplias y que incluyan alumnos de distintas facultades de nuestro país pueden ayudar a ampliar estas conclusiones. Así mismo, estudios que evalúen la actitud antes y después de la enseñanza de la asignatura de psiquiatría también pueden ser útiles para profundizar en esta investigación.

Bibliografía

1. Byrne P. Stigma of mental illness: changing minds, changing behavior. Br J Psychiatry 1999; 174: 1-2.

2. Thornicroft G, Rose D, Kassam A. Discrimination in health care against people with mental illness. Int Rev Psychiatry 2007; 19: 113-22

3. Wolff G, Pathare S, Craig T, Leff J. Community knowledge of mental illness and reaction to mentally ill people. Br J Psychiatry 1996; 168: 183-90.

4. Corrigan PW, Green A, Lundin R, Kubiak M, Penn DL. 
Familiarity with a social distance from people who have serious mental illness. Psychiatr Serv 2001; 52: 953-8.

5. Crisp AH, Gelder M, Goddard E, Meltzer H. Stigmatisation of people with mental illness: a follow-up study within the Changing Minds campaign of the Royal College of Psychiatrists. World Psychiatry 2005; 4: 106-13.

6. Markstrom U, Gyllesten A, Bejerholm U, Bjorkman T, Brunt D, Harrison L, et al. Attitudes towards mental illness among health care students at Swedish universities -a follow up study after completed clinical placement. Nurs Educ Today 2009; 29: 660-5.

7. Hayman-White K, Happell B. Nursing students' attitudes toward mental health nursing and consumers: psychometric properties of a self-report scale. Arch Psychiatr Nurs 2005; 19: 184-93.

8. Aghukwa CN. Medical students' beliefs and attitudes toward mental illness: effects of a psychiatric education. Acad Psychiatry 2010; 34: 67-70.

9. Singh SP, Baxter H, Standen P, Duggan C. Changing the attitudes of 'tomorrow's doctors' towards mental illness and psychiatry: a comparison of two teaching methods. Med Educ 1998; 32: 115-20.

10. Balon R, Franchini GR, Freeman PS, Hassenfeld IN, Keshavan MS, Yoder E. Medical students' attitudes and views of psychiatry. Acad Psychiatry 1999; 23: 30-6.

11. Pailhez G, Bulbena A, Bulbena-Cabré A, Guilera C, Ventura J. Cambios en las actitudes vocacionales hacia la psiquiatría de los estudiantes de medicina durante la carrera. Archivos de Psiquiatría 2008; 2-4: 96-108.

12. Corrigan PW, Waltson AC. The stigma of psychiatric disorders and the gender, ethnicity and education of the perceiver. Community Ment Health J 2007; 43: 439-58.

13. Corrigan PW, River LP, Lundin RK, Penn DL, Uphoff-
Wasowski K, Campion J. Three strategies for changing attributions about severe mental illness. Schizophr Bull 2001; 27: $187-95$

14. Reddy JP, Tan SMK, Azmi MT, Shaharom MH, Rosdinom R, Maniam T, et al. The effect of a clinical posting in psychiatry on the attitudes of medical students towards psychiatry and mental illness in a Malaysian medical school. Ann Acad Med Singapore 2005; 34: 505-10.

15. Schieman S, Van Gundy K. The personal and social links between age and self-reported empathy. Soc Psychol Q 2000; 63: 152-74.

16. Holmes EP, Corrigan PW, Williams P, Canar J, Kubiak MA. Changing public attitudes about schizophrenia. Schizophr Bull 1999; 25: 447-56.

17. Ay P, Save D, Fidanoglu O. Does stigma concerning mental disorders differ through medical education? A survey among medical students in Istanbul. Soc Psychiatry Psychiatr Epidemiol 2006; 41: 63-7.

18. Crespo M, Pérez-Santos E, Muñoz M, Guillén AI. Descriptive study of stigma associated with severe and persistent mental illness among the general population of Madrid (Spain). Community Ment Health J 2008; 44: 393-403.

19. Mukherjee R, Fialho A, Wijetunge A, Checinski K, Surgenor T. The stigmatization of psychiatric illness: the attitudes of medical students and doctors in a London teaching hospital. Psychiatr Bull 2002; 26: 178-81.

20. Galletly $\mathrm{CH}$, Burton C. Improving medical student attitudes towards people with schizophrenia. Aust N Z J Psychiatry 2011; 45: 473-6.

21. Pailhez-Vindual G, Martín-López LM, Rosado-Figuerola S, Bulbena-Vilarrasa A. Perspectivas hacia la psiquiatría de los estudiantes de medicina ocho años después. Psiquiatr Biol 2011; 18: 1-5. 